\title{
Electromagnetic response of the gauge-field model
}

\author{
David M. T. Kuo and C. D. Hu \\ Department of Physics, National Taiwan University, Taipei Taiwan, Republic of China
}

(Received 15 November 1995; revised manuscript received 18 June 1996)

\begin{abstract}
We calculated the polarization function of the gauge field model at finite temperature. The effect of the gauge field was considered to the order of $\mathrm{O}^{0}(N)$ in the convention of a $1 / N$ expansion. We found significant cancellation among Feynman diagrams. As a result, the real part of conductivity has the following behavior: In the low frequency region it is proportional to $1 / \omega^{2}$, similar to that in the Drude model and in the infraredfrequency region there is contribution from an exciting gauge field and particle-hole pair. This makes $\operatorname{Re} \sigma(\omega)$ decrease much slower than that in the Drude model. Another important consequence of the cancellation is that $\operatorname{Re} \sigma(\omega)$ in the low-frequency region is temperature sensitive but not so when the frequency approaches the Fermi energy. [S0163-1829(96)02245-X]
\end{abstract}

\section{INTRODUCTION}

In the past few years the gauge field model has been studied extensively. ${ }^{1-5}$ It is possible that this model contains the most important physics of high-temperature superconductors (HTSC's). The temperature dependence of the resistivity of HTSC's can be explained by a gauge field model ${ }^{2}$ though there is problem with the Hall coefficient. The infrared reflection spectrum ${ }^{6,7}$ of the normal state of the untwined HTCS samples shows that the real part of the optical conductivity is proportional to $1 / \omega^{i}$ where $i$ is close to 1 . This non-Drude behavior may be an indication that HTCS's are not a conventional Fermi liquid. Kim et al. ${ }^{5}$ studied the optical conductivity of fermions interacting with gauge fields and found that the gauge field has a profound effect on the optical conductivity. Thus it is interesting to see if the gauge model can produce a result which is in accordance with experimental results. Our work is an attempt to clarify if it can give a correct description of HTCS's. Since we were concerned with the normal state behavior, we do the calculation at finite temperature.

In the continuum limit, the gauge field has the Lagrangian density

$$
L=f^{*} \partial_{\tau} f+b^{*} \partial_{\tau} b-\frac{1}{2 m_{F}} f^{*} \nabla^{2} f-\frac{1}{2 m_{B}} b^{*} \nabla^{2} b+\widetilde{L},
$$

where

$\widetilde{L}=-\mathbf{j}_{F} \cdot \mathbf{a}-\mathbf{j}_{B} \cdot\left(\mathbf{a}+\frac{e}{c} \mathbf{A}\right)+\frac{1}{2 m_{F}} \rho_{F} \mathbf{a}^{2}+\frac{1}{2 m_{B}} \rho_{B}\left(\mathbf{a}+\frac{e}{c} \mathbf{A}\right)^{2}$,

where $m_{F(B)}$ denotes the mass of fermions (bosons) and $\mathbf{A}$ and $\mathbf{a}$ are the external and gauge fields, respectively. The Coulomb gauge was chosen so that the time components $a_{0}$ and $A_{0}$ vanish. The effective Lagrangian density in Eq. (1) gives a picture of fermions and bosons interacting with a gauge field. This description was introduced by Baskaran and Anderson. ${ }^{8}$ The gauge field was discussed in details by Ioffe and Larkin. ${ }^{9}$ Recently other authors ${ }^{2,10}$ derived similar results using a slave boson formulation ${ }^{11}$ in the $t-J$ model. We start with Eq. (1) and derive the form of the polarization function to second order in the gauge field in Sec. II. In Sec. III we analyze the behavior of the response function. The numerical results are given in Sec. IV with discussions and a comparison with experimental data. Section V is the conclusion.

\section{FORMALISM}

There are two components in the system, fermions and bosons. The physical polarization $\Pi_{e}$ of system is given by

$$
\Pi_{e}=\frac{\Pi_{F} \Pi_{B}}{\Pi_{F}+\Pi_{B}}
$$

which was obtained first by Ioffe and Larkin. ${ }^{9} \Pi_{F}$ and $\Pi_{B}$ are, respectively, the polarization of the fermions and bosons under a vector field. Therefore, they are tensors. We are interested in the optical conductivity of system. It is related to the $x-x$ component of polarization:

$$
\operatorname{Re} \sigma(\Omega, Q \rightarrow 0)=\frac{e^{2}}{\Omega} \operatorname{Im} \Pi_{e, x x}(\Omega, Q \rightarrow 0) .
$$

Since the real part of the optical conductivity is physically more interesting, we shall concentrate our attention on the imaginary part of $\Pi_{e}(\Omega, Q)$. Altshuler, Ioffe, and Millis ${ }^{12}$ have shown that $(1 / N)$ expansion is the only reasonable pertubation in this two-dimensional system with a singular gauge interaction. Hence, following the $1 / N$ expansion approach we calculate all diagrams in Figs. 1 and 2 which are of order $N$ and $(1 / N)^{0}$, respectively. In Fig. 2 each gauge field denoted by a dashed line is proportional to $1 / N$ and a closed particle loop is proportional to $N$. It is hoped that we

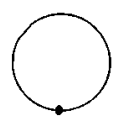

(a)

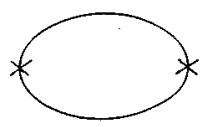

(b)
FIG. 1. The polarization of $\Pi$ of free particles: (a) diamagnetic diagram, (b) paramagnetic diagram. 


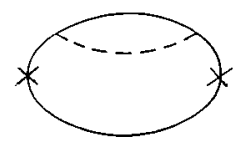

(a) (b)
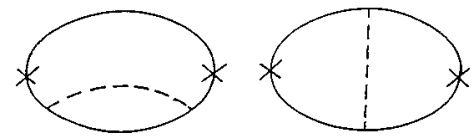

(c)

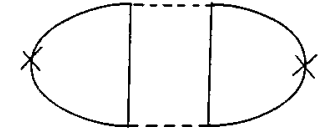

(d)

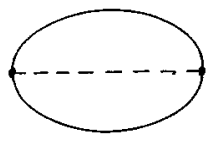

(f)

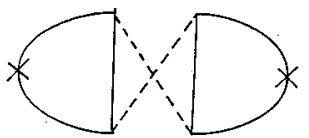

(e)

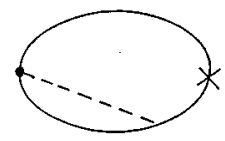

$(9)$
FIG. 2. $(1 / N)^{0}$ correction to the polarization. Crosses denote current vertices and dots denote density vertices.

can probe deeper into the properties of the gauge field and obtain results which are instructive and comparable with experimental data.

We calculated the polarization of fermions first and show later that bosons has a similar form. The free fermion polarization is shown in Fig. 1 and has the following form:

$$
\Pi_{F, i j}^{0}(Q, \Omega)=-\frac{n_{F}}{m_{F}}+2 \int \frac{d^{2} \mathbf{p}}{4 \pi^{2}} \frac{p_{i} p_{j}}{m_{F}^{2}} \frac{f\left(\boldsymbol{\epsilon}_{\mathbf{p}+\mathbf{q}}\right)-f\left(\epsilon_{\mathbf{p}}\right)}{\Omega+\epsilon_{\mathbf{p}}-\epsilon_{\mathbf{p}+\mathbf{q}}+i \delta},
$$

where $n_{F}$ is the number density of the fermions. $f\left(\epsilon_{\mathbf{p}}\right)$ is the Fermi distribution function, $\delta$ is a positive infinitesimal value, $i$ and $j$ are the indices of direction, and the factor of 2 is the spin multiplicity ( $N=2$ in the $1 / N$ expansion). In the frequency region $\Omega>v_{F} q$ where $v_{F}$ is the Fermi velocity, the imaginary part of $\Pi_{F}^{0}(Q, \Omega)$ vanishes because this regime is outside of the particle-hole excitation continuum. Hence it contributes to the imaginary part of conductivity only. The real part of conductivity can be obtained from the next-order diagrams shown in Fig. 2. The first two diagrams are the polarizations with self-energy and the third has a vertex. They, combined, satisfy one of Ward's identities. The dashed line in Fig. 2 is the gauge field which has the structure

$$
\begin{aligned}
D_{i j}(q, \omega) & =\left(\delta_{i j}-\frac{q_{i} q_{j}}{q^{2}}\right) d(q, \omega) \\
& =\left(\delta_{i j}-\frac{q_{i} q_{j}}{q^{2}}\right) \frac{1}{\Pi_{F, x x}^{0}(q, \omega)+\Pi_{B, x x}^{0}(q, \omega)} .
\end{aligned}
$$

The gauge field is considered in the random phase approximation (RPA) level. Higher-order terms are negligible in the low-frequency regime. ${ }^{13}$ Since we consider only the range where photon energy is less than the Fermi energy and the major contribution of the guage field comes from the lowfrequency domain ${ }^{2,6}$ the RPA is adequate for our purpose.

Using Feynman rules ${ }^{14}$ the expressions of diagrams in the Figs. 2(a) and 2(b) are given by

$$
\begin{aligned}
\Pi_{F, i j}^{1 a}\left(Q, i \omega_{n}\right)= & 2 T \sum_{\mathbf{p}, l} \frac{\left(2 p_{i}+Q_{i}\right)\left(2 p_{j}+Q_{j}\right)}{4 m_{F}^{2}} \\
& \times\left[G^{0}\left(\mathbf{p}+\mathbf{Q}, \omega_{l+n}\right)\right]^{2} \\
& \times S\left(\mathbf{p}+\mathbf{Q}, \omega_{l+n}\right) G^{0}\left(\mathbf{p}, \omega_{l}\right)
\end{aligned}
$$

and

$$
\begin{aligned}
& \Pi_{F, i j}^{1 b}\left(Q, i \omega_{n}\right)= 2 T \sum_{\mathbf{p}, l} \frac{\left(2 p_{i}+Q_{i}\right)\left(2 p_{j}+Q_{j}\right)}{4 m_{F}^{2}} \\
& \times\left[G^{0}\left(\mathbf{p}, \omega_{l-n}\right)\right]^{2} S\left(\mathbf{p}, \omega_{l-n}\right) G^{0}\left(\mathbf{p}+\mathbf{Q}, \omega_{l}\right) . \\
& G^{0}\left(\mathbf{p}, i \omega_{l}\right)=\frac{1}{\left(i \omega_{l}-\epsilon_{\mathbf{p}}+\mu_{F}\right)}
\end{aligned}
$$

is the free fermion Green's function, $\mu_{F}$ is the chemical potential of fermions, and

$$
S\left(\mathbf{q}, \omega_{n}\right)=T \sum_{\mathbf{p}, l} \frac{p_{i} p_{j}}{m_{F}^{2}} D_{i j}\left(\mathbf{p}+\mathbf{q}, \omega_{n+l}\right) G^{0}\left(\mathbf{p}, \omega_{l}\right)
$$

is the self-energy. For optical conductivity $Q$, the momentum of the external field can be set to zero. Therefore

$$
\begin{aligned}
\Pi_{F, i j}^{1 a}\left(0, i \omega_{n}\right)+\Pi_{F, i j}^{1 b}\left(0, i \omega_{n}\right)= & \frac{2 T}{i \omega_{n}} \sum_{\mathbf{p}, l} \frac{p_{i} p_{j}}{m_{F}^{2}} \\
& \times\left[S\left(\mathbf{p}, \omega_{l+n}\right)-S\left(\mathbf{p}, \omega_{l}\right)\right] \\
& \times G^{0}\left(\mathbf{p}, \omega_{l+n}\right) G^{0}\left(\mathbf{p}, \omega_{l}\right) .
\end{aligned}
$$

The expression of the diagram in Fig. 2(c) is given by

$$
\begin{aligned}
\Pi_{F, i j}^{1 c}\left(Q, i \omega_{n}\right)= & 2 T \sum_{\mathbf{p}, l} \frac{2 p_{i}+Q_{i}}{2 m_{F}} \Gamma_{j}\left(\mathbf{p}, \mathbf{Q} ; i \omega_{l}, i \omega_{n}\right) \\
& \times G^{0}\left(\mathbf{p}+\mathbf{Q}, \omega_{n+l}\right) G^{0}\left(\mathbf{p}, \omega_{l}\right),
\end{aligned}
$$

where the vertex function

$$
\begin{aligned}
\Gamma_{j}\left(\mathbf{p}, \mathbf{Q} ; i \omega_{l}, i \omega_{n}\right)= & T \sum_{\mathbf{q}, m, h, k} \frac{\left(2 p_{j}+2 q_{j}+Q_{j}\right)}{2 m_{F}} \frac{\left(p_{h}+Q_{h}\right) p_{k}}{m_{F}^{2}} \\
& \times D_{h k}\left(q, i \omega_{m}\right) G^{0}\left(\mathbf{p}+\mathbf{q}, \omega_{l+m}\right) \\
& \times G^{0}\left(\mathbf{p}+\mathbf{q}+\mathbf{Q}, \omega_{l+n+m}\right)
\end{aligned}
$$

Combining Eqs. (11) and (12) at $Q=0$, we obtain the firstorder correction of the gauge field to the polarization of fermions at the long-wavelength limit:

$$
\begin{aligned}
\Pi_{F, i j}^{1}\left(i \omega_{n}\right)= & 2 T^{2} \sum_{\mathbf{p}, \mathbf{q}, m, l, h, k} \frac{q_{i} p_{j}}{m_{F}^{2}} \frac{p_{h} p_{k}}{m_{F}^{2}}\left(\delta_{h k}-\frac{q_{h} q_{k}}{q^{2}}\right) \\
& \times d\left(q, i \omega_{m}\right) h\left(\mathbf{p}, \mathbf{q}, i \omega_{l}, i \omega_{m}, i \omega_{n}\right),
\end{aligned}
$$

where 


$$
\begin{aligned}
h\left(\mathbf{p}, \mathbf{q}, i \omega_{l}, i \omega_{m}, i \omega_{n}\right)= & \frac{1}{i \omega_{l+m}-\epsilon_{\mathbf{p}+\mathbf{q}}+\mu_{F}} \\
& \times \frac{1}{i \omega_{l+n+m}-\epsilon_{\mathbf{p}+\mathbf{q}}+\mu_{F}} \\
& \times \frac{1}{i \omega_{l+n}-\epsilon_{\mathbf{p}}+\mu_{F}} \\
& \times \frac{1}{i \omega_{l}-\epsilon_{\mathbf{p}}+\mu_{F}} .
\end{aligned}
$$

Working out the contour integrals we get

$$
\begin{array}{rl}
T \sum_{l} & h\left(\mathbf{p}, \mathbf{q}, i \omega_{l}, i \omega_{m}, i \omega_{n}\right) \\
= & \frac{f\left(\boldsymbol{\epsilon}_{\mathbf{p}}\right)-f\left(\boldsymbol{\epsilon}_{\mathbf{p}+\mathbf{q}}\right)}{\left(i \omega_{n}\right)^{2}}\left[\frac{2}{i \omega_{m}+\epsilon_{\mathbf{p}}-\epsilon_{\mathbf{p}+\mathbf{q}}}\right. \\
& \left.-\frac{1}{i \omega_{n+m}+\epsilon_{\mathbf{p}}-\epsilon_{\mathbf{p}+\mathbf{q}}}-\frac{1}{i \omega_{m-n}+\epsilon_{\mathbf{p}}-\epsilon_{\mathbf{p}+\mathbf{q}}}\right] .
\end{array}
$$

Inserting Eq. (16) into Eq. (14), we obtain

$$
\Pi_{F}^{1}\left(i \omega_{n}\right)=\Pi_{F}^{11}\left(i \omega_{n}\right)+\Pi_{F}^{12}\left(i \omega_{n}\right)+\Pi_{F}^{13}\left(i \omega_{n}\right),
$$

where

$$
\begin{array}{r}
\Pi_{F, i j}^{11}\left(i \omega_{n}\right) \\
=2 T \sum_{p, q, m} \frac{q_{i} p_{j}}{m_{F}^{2}}\left|\frac{\mathbf{p} \times \hat{\mathbf{q}}}{m_{F}}\right|^{2} \\
\quad \times \frac{d\left(q, i \omega_{m}\right)}{\omega_{n}^{2}} \frac{f\left(\boldsymbol{\epsilon}_{\mathbf{p}}\right)-f\left(\boldsymbol{\epsilon}_{\mathbf{p}+\mathbf{q}}\right)}{i \omega_{n+m}+\boldsymbol{\epsilon}_{\mathbf{p}}-\boldsymbol{\epsilon}_{\mathbf{p}+\mathbf{q}}}, \\
\Pi_{F, i j}^{12}\left(i \omega_{n}\right) \\
=2 T \sum_{p, q, m} \frac{q_{i} p_{j}}{m_{F}^{2}}\left|\frac{\mathbf{p} \times \hat{\mathbf{q}}}{m_{F}}\right|^{2} \\
\quad \times \frac{d\left(q, i \omega_{m}\right)}{\omega_{n}^{2}} \frac{f\left(\boldsymbol{\epsilon}_{\mathbf{p}}\right)-f\left(\boldsymbol{\epsilon}_{\mathbf{p}+\mathbf{q}}\right)}{i \omega_{m-n}+\boldsymbol{\epsilon}_{\mathbf{p}}-\boldsymbol{\epsilon}_{\mathbf{p}+\mathbf{q}}},
\end{array}
$$

and

$$
\begin{aligned}
\Pi_{F, i j}^{13}( & \left(i \omega_{n}\right) \\
= & -4 T \sum_{p, q, m} \frac{q_{i} p_{j}}{m_{F}^{2}}\left|\frac{\mathbf{p} \times \hat{\mathbf{q}}}{m_{F}}\right|^{2} \\
& \times \frac{d\left(q, i \omega_{m}\right)}{\omega_{n}^{2}} \frac{f\left(\boldsymbol{\epsilon}_{\mathbf{p}}\right)-f\left(\boldsymbol{\epsilon}_{\mathbf{p}+\mathbf{q}}\right)}{i \omega_{m}+\boldsymbol{\epsilon}_{\mathbf{p}}-\boldsymbol{\epsilon}_{\mathbf{p}+\mathbf{q}}},
\end{aligned}
$$

where $\hat{\mathbf{q}}$ is a unit vector. With variable changes $\omega_{m} \rightarrow-\omega_{m}$ and $\mathbf{p}+\mathbf{q} \rightarrow-\mathbf{p}$, we find

$$
\begin{aligned}
\Pi_{F, i j}^{1}\left(i \omega_{n}\right)= & \frac{T}{\omega_{n}^{2}} \sum_{\mathbf{p}, \mathbf{q}, m} \frac{q_{i} q_{j}}{m_{F}^{2}} d\left(q, i \omega_{m}\right) \\
& \times\left[P_{F}^{C}\left(q, i \omega_{m+n}\right)-P_{F}^{C}\left(q, i \omega_{m}\right)\right],
\end{aligned}
$$

where

$$
\begin{aligned}
P_{F}^{C}\left(q, i \omega_{n}\right) & =\frac{n_{F}}{m_{F}}+\Pi_{F}^{0}(q, i \omega) \\
& =2 \sum_{\mathbf{p}}\left|\frac{\mathbf{p} \times \hat{\mathbf{q}}}{m_{F}}\right|^{2} \frac{f\left(\boldsymbol{\epsilon}_{\mathbf{p}+\mathbf{q}}\right)-f\left(\boldsymbol{\epsilon}_{\mathbf{p}}\right)}{i \omega_{n}+\epsilon_{\mathbf{p}}-\boldsymbol{\epsilon}_{\mathbf{p}+\mathbf{q}}} .
\end{aligned}
$$

For later use, we also defined that of bosons (subscript $B$ ),

$$
\begin{aligned}
P_{B}^{C}\left(q, i \omega_{n}\right) & =\frac{n_{B}}{m_{B}}+\Pi_{B}^{0}(q, i \omega) \\
& =\sum_{\mathbf{p}}\left|\frac{\mathbf{p} \times \hat{\mathbf{q}}}{m_{B}}\right|^{2} \frac{b\left(\boldsymbol{\epsilon}_{\mathbf{p}+\mathbf{q}}\right)-b\left(\boldsymbol{\epsilon}_{\mathbf{p}}\right)}{i \omega_{n}+\boldsymbol{\epsilon}_{\mathbf{p}}-\boldsymbol{\epsilon}_{\mathbf{p}+\mathbf{q}}},
\end{aligned}
$$

where $b\left(\epsilon_{\mathbf{p}}\right)$ is the Bose-Einstein distribution function. Equation (21) can be changed into

$$
\begin{aligned}
\Pi_{F, i j}^{1}\left(i \omega_{n}\right) & \\
= & \frac{T}{2 \omega_{n}^{2}} \sum_{m, \mathbf{q}} \frac{q_{i} q_{j}}{m_{F}^{2}}\left[d\left(q, i \omega_{m}\right)-d\left(q, i \omega_{m+n}\right)\right] \\
& \times\left[P_{F}^{C}\left(q, i \omega_{m+n}\right)-P_{F}^{C}\left(q, i \omega_{m}\right)\right]
\end{aligned}
$$

for later purposes. The sum of diagrams of Figs. 2(d) and $2(\mathrm{e})$ is equal to

$$
\begin{aligned}
\Pi_{F, i j}^{2}\left(i \omega_{n}\right)= & -\frac{T}{2 \omega_{n}^{2}} \sum \frac{q_{i} q_{j}}{m_{F}^{2}} d\left(q, i \omega_{m}\right) d\left(q, i \omega_{m+n}\right) \\
& \times\left[P_{F}^{C}\left(q, i \omega_{m+n}\right)-P_{F}^{C}\left(q, i \omega_{m}\right)\right]^{2} .
\end{aligned}
$$

In view of Eqs. (5) and (6), we get a significant cancellation between Eqs. (24) and (25):

$$
\begin{aligned}
\Pi_{F, i j}^{C}\left(i \omega_{n}\right)= & \Pi_{F, i j}^{1}\left(i \omega_{n}\right)+\Pi_{F, i j}^{2}\left(i \omega_{n}\right) \\
= & \frac{T}{2 \omega_{n}^{2}} \sum \frac{q_{i} q_{j}}{m_{F}^{2}} d\left(q, i \omega_{m}\right) d\left(q, i \omega_{m+n}\right) \\
& \times\left[P_{F}^{C}\left(q, i \omega_{m+n}\right)-P_{F}^{C}\left(q, i \omega_{m}\right)\right] \\
& \times\left[P_{B}^{C}\left(q, i \omega_{m+n}\right)-P_{B}^{C}\left(q, i \omega_{m}\right)\right] .
\end{aligned}
$$

The superscript $C$ stands for the current polarization. Analogously the boson part can be obtained by replacing $m_{F}$ with $m_{B}:$

$$
\begin{aligned}
\Pi_{B, i j}^{C}\left(i \omega_{n}\right)= & \frac{T}{2 \omega_{n}^{2}} \sum_{m, \mathbf{q}} \frac{q_{i} q_{j}}{m_{B}^{2}} d\left(q, i \omega_{m}\right) d\left(q, i \omega_{m+n}\right) \\
& \times\left[P_{F}^{C}\left(q, i \omega_{m+n}\right)-P_{F}^{C}\left(q, i \omega_{m}\right)\right] \\
& \times\left[P_{B}^{C}\left(q, i \omega_{m+n}\right)-P_{B}^{C}\left(q, i \omega_{m}\right)\right] .
\end{aligned}
$$

The above cancellation shows that one has to take into account appropriate diagrams in order to get reasonable results. Equations (26) and (27) illustrate important features of the gauge field model in that fermions and bosons are on equal footing and interacting strongly with each other. One can no longer distinguish between the contributions from fermions and bosons to physical quantities. 
Finally, the diagram of Fig. 2(g) vanishes because it changes sign under inversion operation. The diagram of Fig. 2(f) with density vertices is equal to

$$
\Pi_{F(B)}^{D}\left(i \omega_{n}\right)=\frac{T}{m_{F(B)}^{2}} \sum_{m, \mathbf{q}} P_{F(B)}^{D}\left(\mathbf{q}, i \omega_{n}+i \omega_{m}\right) d\left(\mathbf{q}, i \omega_{m}\right),
$$

where

$$
P_{F}^{D}\left(\mathbf{q}, i \omega_{n}\right)=2 \sum_{\mathbf{p}} \frac{f\left(\boldsymbol{\epsilon}_{\mathbf{p}+\mathbf{q}}\right)-f\left(\boldsymbol{\epsilon}_{\mathbf{p}}\right)}{i \omega_{n}+\epsilon_{\mathbf{p}}-\epsilon_{\mathbf{p}+\mathbf{q}}}
$$

and

$$
P_{B}^{D}\left(\mathbf{q}, i \omega_{n}\right)=\sum_{\mathbf{p}} \frac{b\left(\epsilon_{\mathbf{p}+\mathbf{q}}\right)-b\left(\epsilon_{\mathbf{p}}\right)}{i \omega_{n}+\epsilon_{\mathbf{p}}-\epsilon_{\mathbf{p}+\mathbf{q}}}
$$

are the density response functions of fermions and bosons and the superscrpt $D$ stands for density. Performing the frequency sum $m$ and analytic continuation $i \omega_{n} \rightarrow \Omega+i \delta$, we obtain the imaginary part of $\Pi_{F(B)}^{C}(\Omega)$ and $\Pi_{F(B)}^{D}(\Omega)$ :

$$
\begin{aligned}
& \operatorname{Im} \Pi_{F(B)}^{C}(\Omega)= \frac{1}{2 \pi \Omega^{2}} \sum_{\mathbf{q}} \frac{q_{x} q_{x}}{m_{F(B)}^{2}} \int d \omega\left[\operatorname{coth}\left(\frac{\omega}{2 T}\right)-\operatorname{coth}\left(\frac{\Omega+\omega}{2 T}\right)\right]\left\{\operatorname{Im}\left[d(q, \omega) P_{B}^{C}(q, \omega)\right] \operatorname{Im}\left[d(q, \Omega+\omega) P_{F}^{C}(q, \Omega+\omega)\right]\right. \\
&\left.-\operatorname{Im}\left[d(q, \omega) P_{F}^{C}(q, \omega) P_{B}^{C}(q, \omega)\right] \operatorname{Im}[d(q, \Omega+\omega)]\right\} \\
& \operatorname{Im}_{F(B)}^{D}(\Omega)=\frac{1}{2 \pi m_{F(B)}^{2}} \sum_{\mathbf{q}} \int d \omega\left[\operatorname{coth}\left(\frac{\omega}{2 T}\right)-\operatorname{coth}\left(\frac{\omega+\Omega}{2 T}\right)\right] \operatorname{Im} d(q, \omega) \operatorname{Im} P_{F(B)}^{D}(q, \Omega+\omega)
\end{aligned}
$$

From now on we neglect direction indices. We shall see in Sec. IV that only the imaginary part of $\Pi_{F(B)}^{C}(\Omega)$ and $\Pi_{F(B)}^{D}(\Omega)$ contributes significantly to the total polarization.

\section{ANALYSIS}

In this section we study the behaviors of $\Pi_{F(B)}^{C}(\Omega)$ and $\Pi_{F(B)}^{D}(\Omega)$ in the low- and intermediate-frequency regions. Before doing that we expanded $\operatorname{Im} \Pi_{F(B)}^{C}(\Omega)$ as

$$
\operatorname{Im} \Pi_{F(B)}^{C}(\Omega)=\mathcal{K}_{1, F(B)}(\Omega)+\mathcal{K}_{2, F(B)}(\Omega)+\mathcal{K}_{3, F(B)}(\Omega),
$$

where

$$
\begin{aligned}
\mathcal{K}_{1, F(B)}(\Omega)= & \frac{1}{8 \pi^{2} m_{F(B)}^{2} \Omega^{2}} \int d q q^{3} \int d \omega\left[\operatorname{coth}\left(\frac{\omega}{2 T}\right)-\operatorname{coth}\left(\frac{\omega+\Omega}{2 T}\right)\right]|d(q, \omega) d(q, \omega+\Omega)|^{2} \operatorname{Im} P_{B}^{C}(q, \omega) \operatorname{Im} P_{F}^{C}(q, \Omega+\omega) \\
& \times\left\{\left[\frac{n_{F}}{m_{F}}+\frac{n_{B}}{m_{B}}-\operatorname{Re} P_{F}^{C}(q, \omega)-\operatorname{Re} P_{B}^{C}(q, \Omega+\omega)\right]^{2}+\left[\operatorname{Im} P_{F}^{C}(q, \omega)+\operatorname{Im} P_{B}^{C}(q, \Omega+\omega)\right]^{2}\right\}, \\
\mathcal{K}_{2, F(B)}(\Omega)= & \frac{1}{8 \pi^{2} m_{F(B)}^{2} \Omega^{2}} \int d q q^{3} \int d \omega\left[\operatorname{coth}\left(\frac{\omega}{2 T}\right)-\operatorname{coth}\left(\frac{\omega+\Omega}{2 T}\right)\right]|d(q, \omega) d(q, \omega+\Omega)|^{2} \operatorname{Im} P_{B}^{C}(q, \Omega+\omega) \operatorname{Im} P_{B}^{C}(q, \omega) \\
& \times\left\{\operatorname{Re} P_{F}^{C}(q, \omega)\left[\operatorname{Re} P_{F}^{C}(q, \omega)-\operatorname{Re} P_{F}^{C}(q, \Omega+\omega)\right]+\operatorname{Im} P_{F}^{C}(q, \omega)\left[\operatorname{Im} P_{F}^{C}(q, \omega)-\operatorname{Im} P_{F}^{C}(q, \omega+\Omega)\right]\right\},
\end{aligned}
$$

and

$$
\begin{aligned}
\mathcal{K}_{3, F(B)}(\Omega)= & \frac{1}{8 \pi^{2} m_{F(B)}^{2} \Omega^{2}} \int d q q^{3} \int d \omega\left[\operatorname{coth}\left(\frac{\omega}{2 T}\right)-\operatorname{coth}\left(\frac{\omega+\Omega}{2 T}\right)\right]|d(q, \omega) d(q, \omega+\Omega)|^{2} \operatorname{Im} P_{F}^{C}(q, \Omega+\omega) \operatorname{Im} P_{F}^{C}(q, \omega) \\
& \times\left\{\operatorname{Re} P_{B}^{C}(q, \omega)\left[\operatorname{Re} P_{B}^{C}(q, \omega)-\operatorname{Re} P_{B}^{C}(q, \omega+\Omega)\right]+\operatorname{Im} P_{B}^{C}(q, \omega)\left[\operatorname{Im} P_{B}^{C}(q, \omega)-\operatorname{Im} P_{B}^{C}(q, \Omega+\omega)\right]\right\}
\end{aligned}
$$

Among the three $\mathcal{K}_{1}(\Omega)$ is the most important because, as we shall see, the magnitude of boson polarization function is much less than that of fermoions.

Kim et al. ${ }^{5}$ made a detailed analysis of the optical response of a system of fermions interacting with gauge field. They concluded that at $0 \mathrm{~K}$ in the low-frequency limit the imaginary part of polarization is proportional to the frequency to the one-third power in two dimensions. We can make a comparison. However, our system has one important difference; namely, it contains bosons. Not only has the response a boson part, but the gauge field is affected [see Eq. (3)]. Furthermore, we calculated the response at finite temperature which was assumed to be higher than the Bose-Einstein condensation temperature.

For later calculation we derive the forms of current and density response functions for both fermions and bosons: 


$$
\begin{aligned}
& \operatorname{Re} P_{F}^{C}(q, \omega)=\frac{n_{F}}{m_{F}}\left\{1-\frac{2 x_{F}^{2}}{3}-\frac{y_{F}^{2}}{8 x_{F}^{2}}+\operatorname{sgn}\left(x_{F}+\frac{y_{F}}{4 x_{F}}\right) \theta\left(\left(x_{F}+\frac{y_{F}}{4 x_{F}}\right)^{2}-1\right) \frac{\left[\left(x_{F}+y_{F} / 4 x_{F}\right)^{2}-1\right]^{3 / 2}}{3 x_{F}}+\operatorname{sgn}\left(x_{F}-\frac{y_{F}}{4 x_{F}}\right)\right. \\
& \left.\times \theta\left(\left(x_{F}-\frac{y_{F}}{4 x_{F}}\right)^{2}-1\right) \frac{\left[\left(x_{F}-y_{F} / 4 x_{F}\right)^{2}-1\right]^{3 / 2}}{3 x_{F}}\right\}, \\
& \operatorname{Im} P_{F}^{C}(q, \omega)=\frac{n_{F}}{3 m_{F} x_{F}}\left\{\theta\left(1-\left(x_{F}+\frac{y_{F}}{4 x_{F}}\right)^{2}\right)\left[1-\left(x_{F}+\frac{y_{F}}{4 x_{F}}\right)^{2}\right]^{3 / 2}-\theta\left(1-\left(x_{F}-\frac{y_{F}}{4 x_{F}}\right)^{2}\right)\left[1-\left(x_{F}-\frac{y_{F}}{4 x_{F}}\right)^{2}\right]^{3 / 2}\right\}, \\
& \operatorname{Re} P_{B}^{C}(q, \omega)=\frac{n_{B}}{m_{B}}-\frac{k_{B} T}{4 \pi x_{B}}\left[\operatorname{sgn}\left(x_{B}+\frac{y_{B}}{4 x_{B}}\right) \int_{0}^{\left(x_{B}+y_{B} / 4 x_{B}\right)^{2}} \frac{\sqrt{u} d u}{e^{\left(x_{B}+y_{B} / 4 x_{B}\right)^{2}-u-\mu_{B} / k_{B} T}-1}\right. \\
& \left.+\operatorname{sgn}\left(x_{B}-\frac{y_{B}}{4 x_{B}}\right) \int_{0}^{\left(x_{B}-y_{B} / 4 x_{B}\right)^{2}} \frac{\sqrt{u} d u}{e^{\left(x_{B}-y_{B} / 4 x_{B}\right)^{2}-u-\mu_{B} / k_{B} T}-1}\right] \text {, } \\
& \operatorname{Im} P_{B}^{C}(q, \omega)=\frac{\Gamma(3 / 2) k_{B} T}{4 \pi x_{B}} \sum_{n} n^{-3 / 2}\left\{e^{n\left[-\left(x_{B}+y_{B} / 4 x_{B}\right)^{2}+\mu_{B} / k_{B} T\right]}-e^{n\left[-\left(x_{B}-y_{B} / 4 x_{B}\right)^{2}+\mu_{B} / k_{B} T\right]}\right\}, \\
& \operatorname{Re} P_{F}^{D}(q, \omega)=\frac{m_{F}}{2 \pi x_{F}}\left\{x_{F}-\operatorname{sgn}\left(x_{F}+\frac{y_{F}}{4 x_{F}}\right) \theta\left(\left(x_{F}+\frac{y_{F}}{4 x_{F}}\right)^{2}-1\right)\left[\left(x_{F}+\frac{y_{F}}{4 x_{F}}\right)^{2}-1\right]^{1 / 2}-\operatorname{sgn}\left(x_{F}-\frac{y_{F}}{4 x_{F}}\right) \theta\left(\left(x_{F}-\frac{y_{F}}{4 x_{F}}\right)^{2}-1\right)\right. \\
& \left.\times\left[\left(x_{F}-\frac{y_{F}}{4 x_{F}}\right)^{2}-1\right]^{1 / 2}\right\} \\
& \operatorname{Im} P_{F}^{D}(q, \omega)=\frac{m_{F}}{2 \pi x_{F}}\left\{\theta\left(1-\left(x_{F}+\frac{y_{F}}{4 x_{F}}\right)^{2}\right)\left[1-\left(x_{F}+\frac{y_{F}}{4 x_{F}}\right)^{2}\right]^{1 / 2}-\theta\left(1-\left(x_{F}-\frac{y_{F}}{4 x_{F}}\right)^{2}\right)\left[1-\left(x_{F}-\frac{y_{F}}{4 x_{F}}\right)^{2}\right]^{1 / 2}\right\}, \\
& \operatorname{Re} P_{B}^{D}(q, \omega)=\frac{m_{B}}{4 \pi x_{B}}\left\{\operatorname{sgn}\left(x_{B}+\frac{y_{B}}{4 x_{B}}\right) \int_{0}^{\left(x_{B}+y_{B} / 4 x_{B}\right)^{2}} \frac{d u}{\sqrt{u}\left[e^{\left(x_{B}+y_{B} / 4 x_{B}\right)^{2}-u-\mu_{B} / k_{B} T}-1\right]}+\operatorname{sgn}\left(x_{B}-\frac{y_{B}}{4 x_{B}}\right)\right. \\
& \left.\times \int_{0}^{\left(x_{B}-y_{B} / 4 x_{B}\right)^{2}} \frac{d u}{\sqrt{u}\left[e^{\left(x_{B}-y_{B} / 4 x_{B}\right)^{2}-u-\mu_{B} / k_{B} T}-1\right]}\right\},
\end{aligned}
$$

and

$$
\begin{aligned}
\operatorname{Im} P_{B}^{D}(q, \omega)= & \frac{\Gamma(1 / 2) m_{B}}{8 \pi x_{B}} \sum_{n} n^{-1 / 2}\left\{e^{n\left[-\left(x_{B}+y_{B} / 4 x_{B}\right)^{2}+\mu_{B} / k_{B} T\right]}\right. \\
& \left.-e^{n\left[-\left(x_{B}-y_{B} / 4 x_{B}\right)^{2}+\mu_{B} / k_{B} T\right]}\right\}
\end{aligned}
$$

where

$$
\begin{gathered}
x_{F}=\frac{q}{2 k_{F}}, \\
y_{F}=\frac{\omega}{\epsilon_{F}}, \\
x_{B}=\frac{q}{2 \sqrt{2 m_{B} k_{B} T}},
\end{gathered}
$$

and

$$
y_{B}=\frac{\omega}{k_{B} T} .
$$

Though we are interested in the finite-temperature regime, the polarization functions of fermions are derived at $0 \mathrm{~K}$. This is a very good approximation when $k_{B} T \ll \epsilon_{F}$. For the bosons we have use the finite-temperature polarizations. The chemical potential $\mu_{B}$ was evaluated with the of equation free bosons,

$$
n_{B}=\int \frac{d^{2} k}{4 \pi^{2}} b\left(\epsilon_{k}\right),
$$

and was found to be

$$
\mu_{B}=-k_{B} T \exp \left(-\frac{2 \pi n_{B}}{m_{B} k_{B} T}\right),
$$

for $n_{B} / m_{B} \gg k_{B} T$. It turns out that $\mu_{B}$ is negative and very close to 0 . It is of the same order of magnitude even if interaction is considered. ${ }^{16}$ As pointed out by Lee and Nagaosa, ${ }^{2}$ the Bose-Einstein condensation temperature is much greater than the room temperature for HTSC material. One expects the interaction to suppress the condensation temperature. Here, taking the value of the free boson chemical potential value does not necessarily mean the temperature 
range we are interested in is below the realistic condensation temperature. In fact, we believe that a more accurate chemical potential value should give us a much lower condensation temperature but will not affect much our result. Kobuki's calculation $^{15}$ showed that the order of magnitude of the chemical potential is not changed by taking into account the interaction.

Equations (41)-(44) show clearly the momentum and energy scales of fermions and bosons. For $\epsilon_{F}=1 \mathrm{eV}$ at $100 \mathrm{~K}$, we have $\epsilon_{F} / k_{B} T \simeq 100, k_{F} / \sqrt{2 m_{B} T} \simeq 5$, and $m_{F} / m_{B} \simeq 5$ for $J / t \simeq 0.2$. These relations lead to the result that bosons have a smaller energy and momentum scales than those of fermions. Furthermore, the fermion current polarization is much greater in magnitude than that of bosons while the opposite is true for the density polarizations. The polarization function forms of fermions and bosons polarizations are quite similar. The Fermi-Dirac distribution function provides the fermion polarization with a cutoff at $k_{F}$ while the Bose-Einstein distribution has a sharp decrease near $\epsilon_{k}=0$ with a width $k_{B} T$. This analogy made later analysis easier.

By the low-frequency region we mean $\Omega<k_{B} T$. If $\left|\mu_{B}\right|>k_{B} T$, then $\Omega$ is the smallest energy scale and the fermion and boson polarization functions have the similar behavior. According to Kim et al. ${ }^{5}$ the main contribution in the $\omega$ integration comes from the small- $\omega$ region. This can be seen in the following analysis. The common temperature factor in Eqs. (31) and (32) can be approximated by

$$
\operatorname{coth} \frac{\omega}{2 k_{B} T}-\operatorname{coth} \frac{\Omega+\omega}{2 k_{B} T} \simeq \frac{2 k_{B} T \Omega}{\omega(\Omega+\omega)} .
$$

The main contributions to the integrations in Eqs. (31) and (32) come from the small- $q$ and $-\omega$ regions: $y_{F(B)}<1, x_{F(B)}<1$. Here, the polarization functions have the behaviors

$$
\begin{gathered}
\operatorname{Re}_{F}^{C}(\omega) \simeq \frac{n_{F}}{m_{F}}\left(1-\frac{x_{F}^{2}}{3}\right), \\
\operatorname{Im} P_{F}^{C}(\omega) \simeq-\frac{n_{F} y_{F}}{4 m_{F} x_{F}}, \\
\operatorname{Re} P_{B}^{C}(\omega) \simeq \frac{n_{B}}{m_{B}}-\frac{k_{B} T_{B E}^{0} x_{B}^{2}}{3 \pi}, \\
\operatorname{Im} P_{B}^{C}(\omega) \simeq-\frac{k_{B} T y_{B}}{8 \sqrt{\pi} x_{B}}, \\
\operatorname{Im} P_{F}^{D}(\omega) \simeq-\frac{m_{F} y_{F}}{4 \pi x_{F}}, \\
\operatorname{Im} P_{B}^{D}(\omega) \simeq-\frac{m_{B} y_{B}}{8 \sqrt{\pi} x_{B}},
\end{gathered}
$$

and

$$
\frac{1}{d(\omega)} \simeq \frac{x_{F}^{2}}{3}-\frac{k_{B} T_{B E}^{0} x_{B}^{2}}{3 \pi}-i\left(\frac{n_{F} y_{F}}{4 m_{F} x_{F}}+\frac{k_{B} T y_{B}}{8 \sqrt{\pi} x_{B}}\right)
$$

where $T_{B E}^{0}=2 \pi n_{B} / m_{B}$ is the Bose-Einstein condensation temperature of free bosons. Substituting Eqs. (52)-(57) into Eqs. (31)-(36), we find

$$
\begin{aligned}
& \operatorname{Im}_{F(B)}^{C}(\Omega) \propto \frac{1}{\Omega}, \\
& \operatorname{Im}_{F}^{D}(\Omega) \propto \Omega^{2 / 3},
\end{aligned}
$$

and

$$
\operatorname{Im} \Pi_{B}^{D}(\Omega) \propto \Omega .
$$

Note that the $q$ and $\omega$ integrations of $\operatorname{Im}_{B}^{D}(\Omega)$ are bound by $k_{B} T$ while those of $\operatorname{Im}_{F}^{D}(\Omega)$ are not. That is why the frequency dependences are different in Eqs. (60) and (61). Equation (50) is valid for $x_{B}<\left|\mu_{B} / k_{B} T\right|$. However, $\left|\mu_{B}\right| / k_{B} T$ is extremely small even in the systems such as lightly doped HTCS's, for example, 0.1 boson per unit cell and $t=0.5 \mathrm{eV}$. It remains so even if the gauge field contribution is incorporated. ${ }^{15}$ Therefore, in practical systems, the boson polarization function has different behavior. For $x_{B}>\left|\mu_{B} / k_{B} T\right|$ we have

$$
\operatorname{Re} P_{B}^{C}(\Omega) \simeq \frac{k_{B} T}{2 \pi}\left(\ln 4 x_{B}-2\right)
$$

The radius of validity of Eq. (54) is so small that it hardly played any role in the $q$ integration. It is the $\operatorname{Re} P_{B}^{C}(\Omega)$ in Eq. (62) which should be used for analysis. So in view of Eqs. (6), (22), (23), (52), (53), (55)-(57) and (61) we find

$$
\frac{1}{d(q, \omega)} \simeq O\left(\frac{n_{B}}{m_{B}}\right)
$$

and is a slow varying function of $q$ and $\omega$. This form should be used for small $q$ and $\omega$. Putting all the pieces togather, we find that for small frequencies,

$$
\operatorname{Im}_{F(B)}^{D}(\Omega) \propto \Omega
$$

and

$$
\operatorname{Im} \Pi_{F(B)}^{C}(\Omega) \propto \frac{1}{\Omega} .
$$

The experimentally more interesting region is $k_{B} T$ $<\Omega<\epsilon_{F}$. It shall be called the intermediate-frequency region. The fermion polarization functions properties in Eqs. (52)-(57) are still applicable but those of bosons changed drastically:

$$
\begin{gathered}
\operatorname{Im} P_{B}^{C}(\omega) \simeq-k_{B} T\left[\delta\left(y_{B}-4 x_{B}^{2}\right)-\delta\left(y_{B}+4 x_{B}^{2}\right)\right], \\
\operatorname{Im} P_{B}^{D}(\omega) \simeq-\frac{m_{B}}{2 \sqrt{\pi}}\left[\delta\left(y_{B}-4 x_{B}^{2}\right)-\delta\left(y_{B}+4 x_{B}^{2}\right)\right],
\end{gathered}
$$

and $\operatorname{Re} P_{B}^{C}(\Omega)$ becomes very small compared with $n_{B} / m_{B}$. According to above analysis, we found

$$
\frac{1}{|d(\omega, q)|^{2}} \simeq\left(\frac{n_{B}}{m_{B}}\right)^{2}+\left(\frac{\epsilon_{F} y_{F}}{4 \pi x_{F}}\right)^{2}
$$

so that 


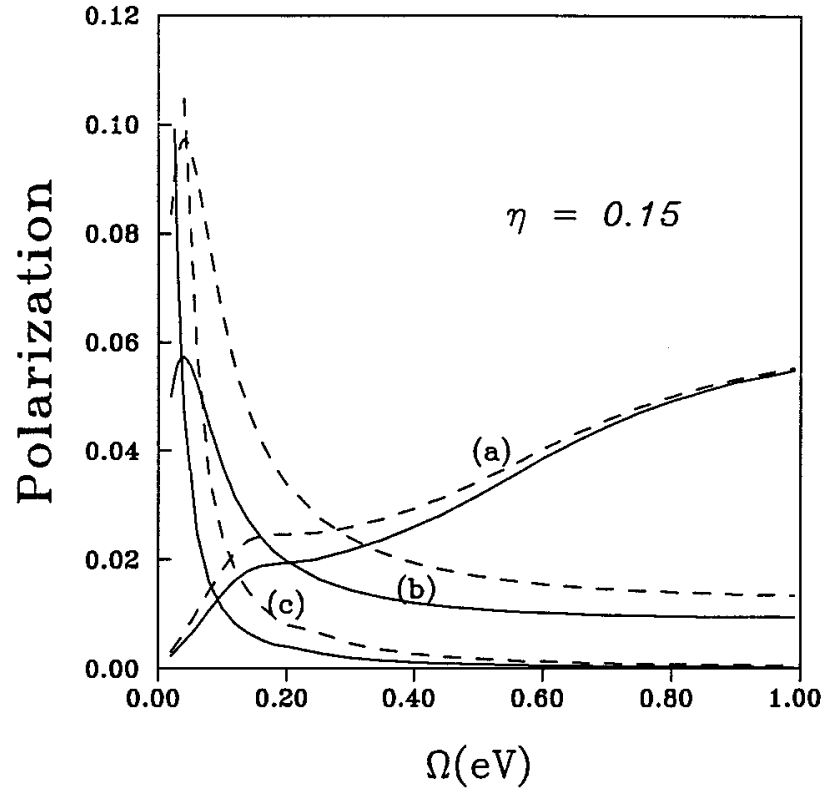

FIG. 3. The imaginary part of polarization functions. The solid lines are those evaluated at $150 \mathrm{~K}$ and dashed lines are those at 200 $\mathrm{K}$. The curves of $\Pi_{F}^{D}(\Omega)$ are denoted by $(\mathrm{a})$, those of $\Pi_{B}^{D}(\Omega)$ are denoted by (b), and those of $\Pi_{B}^{C}(\Omega)$ are denoted by (c).

$$
\begin{gathered}
\operatorname{Im} \Pi_{F(B)}^{C}(\Omega) \propto \frac{1}{\Omega}, \\
\operatorname{Im}_{B}^{D}(\Omega) \propto \frac{\Omega}{c+\Omega^{2}},
\end{gathered}
$$

and

$$
\operatorname{Im} \Pi_{F}^{D}(\Omega) \propto \Omega,
$$

where $c$ is a constant. The whole picture becomes the following: At small frequencies $\operatorname{Im}_{F}^{C}(\Omega)$ and $\operatorname{Im}_{B}^{C}(\Omega)$ dominate but the contribution of $\operatorname{Im}_{F}^{D}(\Omega)$ and $\operatorname{Im}_{B}^{D}(\Omega)$ increases with frequency. As $\Omega$ approaches $\epsilon_{F}, \operatorname{Im}_{F}^{D}(\Omega)$ becomes the most important term.

\section{NUMERICAL RESULTS}

With the conditions of HTCS materials in mind, we compute the polarization numerically. The hopping energy $t$ and the exchange energy $J$ are set to 0.5 and $0.1 \mathrm{eV}$, respectively. The doping concentration is set to be 0.15 in order to simulate low-doping situation. The lattice constant $a=3.8 \AA$ is needed for the fermion and boson mass $m_{F}=1 /\left(2 a^{2} J\right)$ and $m_{B}=1 /\left(2 a^{2} t\right)$. Under above conditions we found that $\epsilon_{F} \simeq 1.1 \mathrm{eV}$.

In Fig. 3 we show the polarizations as functions of frequency. The solid lines are those evaluated at $150 \mathrm{~K}$ and the dashed lines $200 \mathrm{~K}$. Curves (a), (b), and (c) denote $\operatorname{Im} \Pi_{F}^{D}$, $\operatorname{Im} \Pi_{B}^{D}$, and $\operatorname{Im} \Pi_{B}^{C}$. We have not shown $\operatorname{Im} \Pi_{F}^{C}$ since it has the same behavior as $\operatorname{Im} \Pi_{B}^{C}$ but with a smaller magnitude. $\operatorname{Im} \Pi_{F}^{C}(\Omega), \operatorname{Im} \Pi_{B}^{C}(\Omega)$, and $\operatorname{Im} \Pi_{B}^{D}(\Omega)$ contain the free boson polarization function. Hence, curves (b) and (c) show a clear temperature dependence. On the other hand, $\operatorname{Im} \Pi_{F}^{D}$ is not

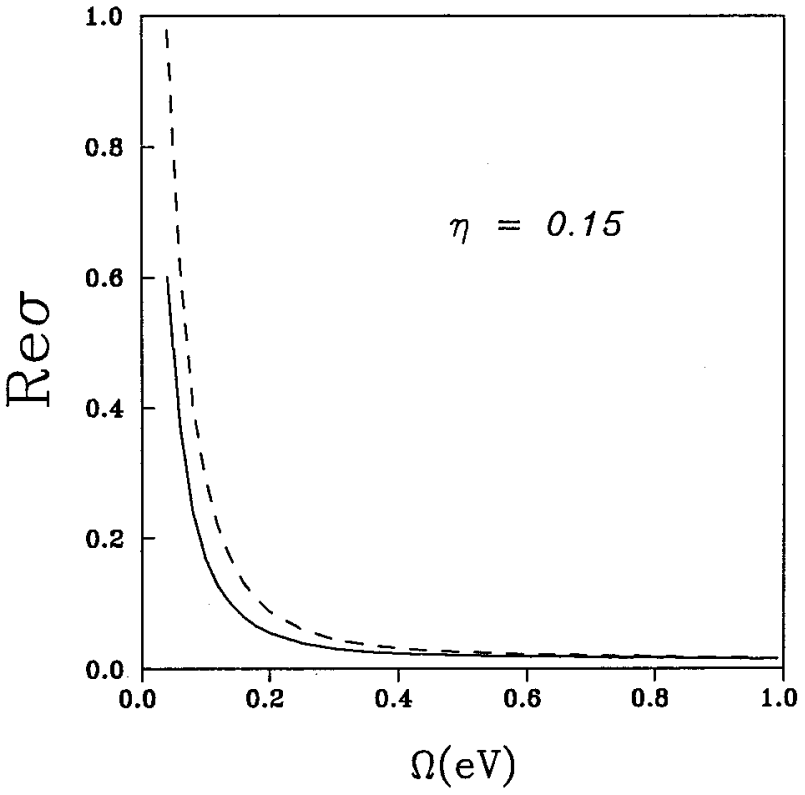

FIG. 4. The real part of conductivity. The solid line is that at $150 \mathrm{~K}$ and dashed lines are that at $200 \mathrm{~K}$.

very sensitive to temperature because it is mainly a fermion effect. The behaviors of these polarizations were analyzed in last section.

We also calculated the response of electrons $\Pi_{e}(\Omega)$ in the intermediate-frequency region. It can be compared with experimental results. According to Eq. (3),

$$
\begin{aligned}
\frac{1}{\Pi_{e}(\Omega)}= & \frac{1}{\Pi_{F}^{0}(\Omega)+\Pi_{F}^{C}(\Omega)+\Pi_{F}^{D}(\Omega)} \\
& +\frac{1}{\Pi_{B}^{0}(\Omega)+\Pi_{B}^{C}(\Omega)+\Pi_{B}^{D}(\Omega)},
\end{aligned}
$$

where

$$
\operatorname{Re}_{F}^{0}(\Omega)=-\frac{n_{F}}{m_{F}}
$$

and

$$
\operatorname{Re}_{B}^{0}(\Omega)=-\frac{n_{B}}{m_{B}}
$$

The imaginary parts of $\Pi_{F}^{0}(\Omega)$ and $\Pi_{B}^{0}(\Omega)$ are vanishingly small if $\Omega>v_{F} Q$ for fermions and $\Omega>\sqrt{2 T / m_{B}} Q$ for bosons. Therefore we can safely neglect them in the intermediate-frequency region. In view of Eq. (3) we can compute the real part of the conductivity. It is plotted in Fig. 4 where the solid line is that at $150 \mathrm{~K}$ and the dashed line is at $200 \mathrm{~K}$. In the frequency range $\Omega<T \sim 0.05 \mathrm{eV}$, it is the contribution of

$$
\Pi_{F}^{C}(\Omega)+\Pi_{B}^{C}(\Omega)
$$

that dominates. Hence it is approximately proportional to $1 / \Omega^{2}$ and it is temperature sensitive. As frequency increases, $\Pi_{B}^{D}(\Omega)$ and $\Pi_{F}^{D}(\Omega)$ become important. In the range 
$\Omega>0.4 \mathrm{eV}, \Pi_{F}^{D}(\Omega)$ provides the largest contribution. The mechanism is that a photon excites a fermion-hole pair and gauge field. Hence, it is not sensitive to a temperature change and it decreases slowly with increasing frequency. The above argument is based on the following analysis:

$$
\operatorname{Im}_{e}=-\frac{B_{1}(\Omega)+B_{2}(\Omega)}{B_{3}(\Omega)},
$$

where

$$
\begin{aligned}
B_{1}(\Omega)= & {\left[\left(\operatorname{Re}_{B}^{0}+\operatorname{Re} \Pi_{B}^{C}+\operatorname{Re} \Pi_{B}^{D}\right)^{2}+\left(\operatorname{Im} \Pi_{B}^{C}+\operatorname{Im} \Pi_{B}^{D}\right)^{2}\right] } \\
& \times\left(\operatorname{Im} \Pi_{F}^{C}+\operatorname{Im} \Pi_{F}^{D}\right) \\
B_{2}(\Omega)= & {\left[\left(\operatorname{Re} \Pi_{F}^{0}+\operatorname{Re} \Pi_{F}^{C}+\operatorname{Re} \Pi_{F}^{D}\right)^{2}+\left(\operatorname{Im} \Pi_{F}^{C}+\operatorname{Im} \Pi_{F}^{D}\right)^{2}\right] } \\
& \times\left(\operatorname{Im} \Pi_{B}^{C}+\operatorname{Im} \Pi_{B}^{D}\right)
\end{aligned}
$$

and

$$
\begin{aligned}
B_{3}(\Omega)= & \left(\operatorname{Re} \Pi_{B}^{0}+\operatorname{Re} \Pi_{B}^{C}+\operatorname{Re} \Pi_{B}^{D}+\operatorname{Re} \Pi_{F}^{0}+\operatorname{Re} \Pi_{F}^{C}+\operatorname{Re} \Pi_{F}^{D}\right)^{2} \\
& +\left(\operatorname{Im} \Pi_{F}^{C}+\operatorname{Im} \Pi_{F}^{D}+\operatorname{Im} \Pi_{B}^{C}+\operatorname{Im} \Pi_{B}^{D}\right)^{2},
\end{aligned}
$$

where we have not written down the argument $\Omega$. $\operatorname{Re} \Pi_{F}^{C}(\Omega), \operatorname{Re} \Pi_{B}^{C}(\Omega), \operatorname{Re} \Pi_{F}^{D}(\Omega)$, and $\operatorname{Re} \Pi_{B}^{D}(\Omega)$ can be neglected because they are much smaller than their zerothorder counterparts. $\operatorname{Im} \Pi_{F(B)}^{C(D)}$ in the denominator is also negligible. Hence, by Eqs. (73) and (74), we found

$\operatorname{Im} \Pi_{e}$

$$
\simeq \frac{\left(n_{F} / m_{F}\right)^{2}\left(\operatorname{Im} \Pi_{B}^{C}+\operatorname{Im} \Pi_{B}^{D}\right)+\left(n_{B} / m_{B}\right)^{2}\left(\operatorname{Im} \Pi_{F}^{C}+\operatorname{Im} \Pi_{F}^{D}\right)}{\left(n_{F} / m_{F}+n_{B} / m_{B}\right)^{2}} .
$$

Now it becomes apparent that $\operatorname{Im} \Pi_{e}$ or $\operatorname{Re} \sigma$ is approximately the linear combination of $\operatorname{Im} \Pi_{B}^{C}+\operatorname{Im} \Pi_{B}^{D}$ and $\operatorname{Im} \Pi_{F}^{C}+\operatorname{Im} \Pi_{F}^{D}$. From Fig. 3, we see that the former dominates in the range $\Omega<0.2 \mathrm{eV}$ and $\operatorname{Im} \Pi_{F}^{D}$ dominates when $\Omega>0.4 \mathrm{eV}$.

Comparing with the Drude model, we found that for $\Omega<T$ the conductivity of the gauge field model has similar frequency dependence. As frequency increases to the order of $T, \operatorname{Im} \Pi_{B}^{D}(\Omega)$ become important and it destroys the Drudelike behavior (see Fig. 3). Hence we can say the conductivity is temperature sensitive in the small- $\Omega$ region. As the frequency increases still further, $\operatorname{Im} \Pi_{F}^{D}(\Omega)$ becomes dominant. The structure in the midinfrared (MIF) region comes from $\Pi_{F}^{D}(\Omega)$. It is responsible for the non-Drude behavior in this region. Bang and Kotliar ${ }^{16}$ had obtained similar non-Drude spectrum for condensated bosons $(T=0)$. From middle infrared $(\mathrm{MIF})$ to $1(\mathrm{eV})$ there is an extra absorption range which is due to what they called convolution-type diagrams, which correspond to our Fig. 2(f). We believe it has the same origin as ours. The reason why the structure in Ref. 16 is more manifest is that their work was on condensated bosons. In our calculation bosons at finite temperature $\left[\Pi_{B}^{D}(\Omega)\right.$ in Fig. 3] provide background which blurred the contribution of $\Pi_{F}^{D}(\Omega)$.

Now we summarize the contributions to optical spectrum in different frequency ranges. In the low-frequency region, here $\Omega<T$, the current-current correlation functions $\Pi_{F(B)}^{C}$ resulting from Figs. 2(a)-2(e) are important and give a Drude-like spectrum. As the frequency $\Omega>T$ the densitydensity correlation functions $\Pi_{B}^{D}$ become significant. As $\Omega>\left(\sqrt{T / \epsilon_{F}}\right)\left(n_{B} / m_{B}\right)$ the fermion contribution $\Pi_{F}^{D}(\Omega)$ dominates. This extra optical absorption in the highfrequency region and Drude-like spectrum in the lowfrequency region were observed. ${ }^{6,7}$

\section{CONCLUSION}

We have studied the properties of the gauge field propagator. They indeed have a profound effect on the optical conductivity of the system. The diagram calculation has been performed up to the $(1 / N)^{0}$ level. We found an important cancellation among the diagrams. This leads to the observation that fermions and bosons are strongly correlated. As a result the current polarization contains a product of a free fermion polarization and a free boson polarization. Due to the presence of the latter, the real part of the conductivity is temperature sensitive if the frequency is less than $\left(\sqrt{T / \epsilon_{F}}\right)$ $\times\left(n_{B} / m_{B}\right)$. When the frequency is greater the fermion density polarization becomes very important. The physical process is a photon excites a particle-hole pair and gauge field simultaneously. We did not persue the frequency region where it is higher than $2 t=1(\mathrm{eV})$. For smaller frequencies $(\Omega<T)$ the real part of the conductivity has a frequency dependence similar to that of the Drude model, but it decreases much slower than that in the Drude model when the frequency is larger. This is the non-Fermi-liquid behavior which is the hallmark of the gauge field model and can be verified by experiments.

\section{ACKNOWLEDGMENTS}

The authors thank P. A. Lee for inspiring discussions. This work was supported in part by the National Science Council of Taiwan, ROC by Grant No. NSC 85-2112-M002-014.
${ }^{1}$ L. B. Ioffe and G. Kotliar, Phys. Rev. B 42, 10348 (1990).

${ }^{2}$ P. A. Lee and N. Nagaosa, Phys. Rev. B 46, 5621 (1992).

${ }^{3}$ J. M. Wheatley and A.J. Schonfield, Phys. Rev. B 47, 11607 (1993)

${ }^{4}$ B. Blok and H. Monien, Phys. Rev. B 47, 3454 (1993).

${ }^{5}$ Y.B. Kim, A.Furusaki, Y. G. Wen, and P. A. Lee, Phys. Rev. B 50, 17917 (1994).
${ }^{6}$ D. B. Tanner et al., in High-Temperature Superconductors, edited by J. Ashkenazi et al. (Plenum, New York, 1991).

${ }^{7}$ Z. Schlesinger et al., Phys. Rev. Lett. 65, 801 (1990).

${ }^{8}$ G. Baskaran and P. W. Andersan, Phys. Rev. B 37, 580 (1988).

${ }^{9}$ L. B. Ioffe and A. Larkin, Phys. Rev. B 39, 8988 (1989).

${ }^{10}$ M. Grilli and G. Kotliar, Phys. Rev. Lett. 64, 1170 (1990).

${ }^{11}$ S. E. Barnes, J. Phys. F 6, 1375 (1976). 
${ }^{12}$ B. L. Altshuler, L. B. Ioffe, and A. J. Millis, Phys. Rev. B 50, 14048 (1994).

${ }^{13}$ Junwu Gan and Eugene Wong, Phys. Rev. Lett. 71, 4226 (1993).

${ }^{14}$ A. A. Abrikosov, L. P. Gor'kov, and I. E. Dzyaloshinski, Meth- ods of Quantum Field Theory in Statistical Physics (PrenticeHall, Englewood Cliffs, NJ, 1963).

${ }^{15}$ K. Kuboki, J. Phys. Soc. Jpn. 62, 420 (1993).

${ }^{16}$ Yunkyu Bang and G. Kotliar, Phys. Rev. B 48, 9898 (1993). 